\title{
Correlative Microscopy in 3D: Recent Advancements in Multi-Scale Materials Science
}

\author{
Jeff Gelb ${ }^{1}$, Tobias Volkenandt ${ }^{2}$ and Arno Merkle ${ }^{1}$ \\ 1. Carl Zeiss Microscopy, Pleasanton, CA, USA. \\ 2. Carl Zeiss Microscopy, Oberkochen, Germany
}

In materials science, the question of scale is of chief importance to any experiment. This may simply be a question of length scale - i.e., what is scale of interesting phenomena? How do features at the millimeter scale relate to features in the nanometer regime? In this case, it may be important to characterize a process at several different magnifications, linking each scale together in compiling the final result. Or, the question may be more complex: for instance, addressing the "multi-scale" challenge may require linking multiple modalities together, integrating spectroscopic data with spatial data, 3D X-ray micrographs with FIB-SEM data volumes. In this case, addressing the question may involve using 3D X-ray data as a survey to probe the large-scale microstructure [1], providing a 3D survey to drive subsequent imaging experiments with the FIB-SEM [2]. In so doing, a "multi-scale" experiment may involve incorporating multiple time points with 4D X-ray microscopy (XRM), or involving collaborators in a multi-user environment.

Multi-scale microscopy, clearly, incorporates a wide range of experimental techniques, a multitude of experimental results, and may involve a variety of researchers, who may be footsteps or oceans apart. Addressing the "multi-scale" challenge is, thus, a challenge unto itself: in order to facilitate multi-scale materials science, a multi-scale approach must be taken, which integrates results from a variety of techniques and data sources, while providing a common workspace to facilitate communication amongst researchers. [3]

Over the past few years, the "multi-scale challenge" has been addressed by both academic and commercial ventures alike. In this presentation, we will explore the recent developments in correlative microscopy as a catalyst for multi-scale materials science, with particular emphasis on the XRM to FIB-SEM workflow. We will present a novel correlative microscopy workspace in which researchers from any background can access correlative microscopy results, combining data from light-, electron-, and X-ray microscopy to produce an integrated dataset richer than any of its components on its own. We will demonstrate how correlative microscopy has been applied in a composite research field, using high-resolution light microscopy for an initial survey, correlative XRM in the same region of interest to build a volumetric representation of the microstructure, and correlative EDS mapping to clarify the local composition. These results were used to build an integrated multi-scale model of the material's structure and composition, which was subsequently used to predict the performance characteristics of the bulk material. We will also review some prior work, where a correlative XRM to FIB-SEM workflow was used to elucidate the multiscale response of a magnesium alloy to a corrosive environment. In the context of that study, 4D XRM was used to track the microstructure evolution of the alloy as it was corroded in situ, followed by correlative high-resolution FIB-SEM imaging to map the finer details of the corroded microstructure.

Correlative microscopy represents an exciting pathway for the future of materials science. Using the unique tools described here, the multi-scale challenge may be sufficiently addressed, increasing research efficiencies and bridging the gaps between researchers of different backgrounds. 


\section{References:}

[1] AP Merkle and J Gelb, Micros. Today. 21 (2013), p. 10-15.

[2] SS Singh, JL Loza, AP Merkle and N Chawla, Materials Characterization 118 (2016), p. 102-111.

[3] AP Merkle, L Lechner, A Steinbach, J Gelb, M Kienle, MW Phaneuf, D Unrau, SS Singh and N Chawla, Microsc. Microanal. (2014), p. S10-S13.
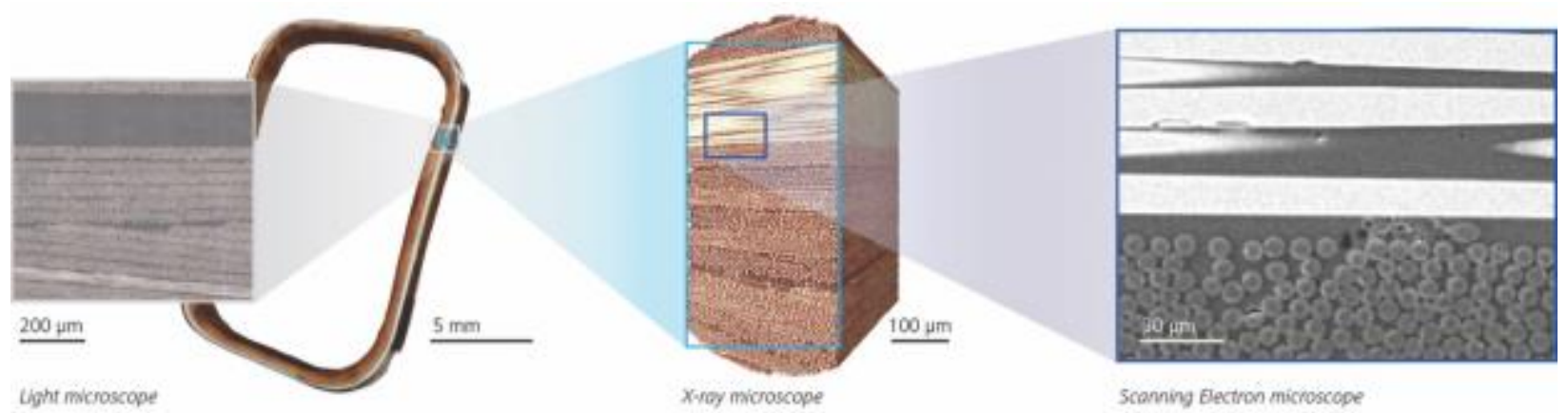

Figure 1. Multi-scale correlative microscopy performed on a carbon-fiber reinforced composite. The study began with light microscopy, continued with 3D X-ray microscopy, and ended with correlative SEM and EDS analysis of a targeted ROI.
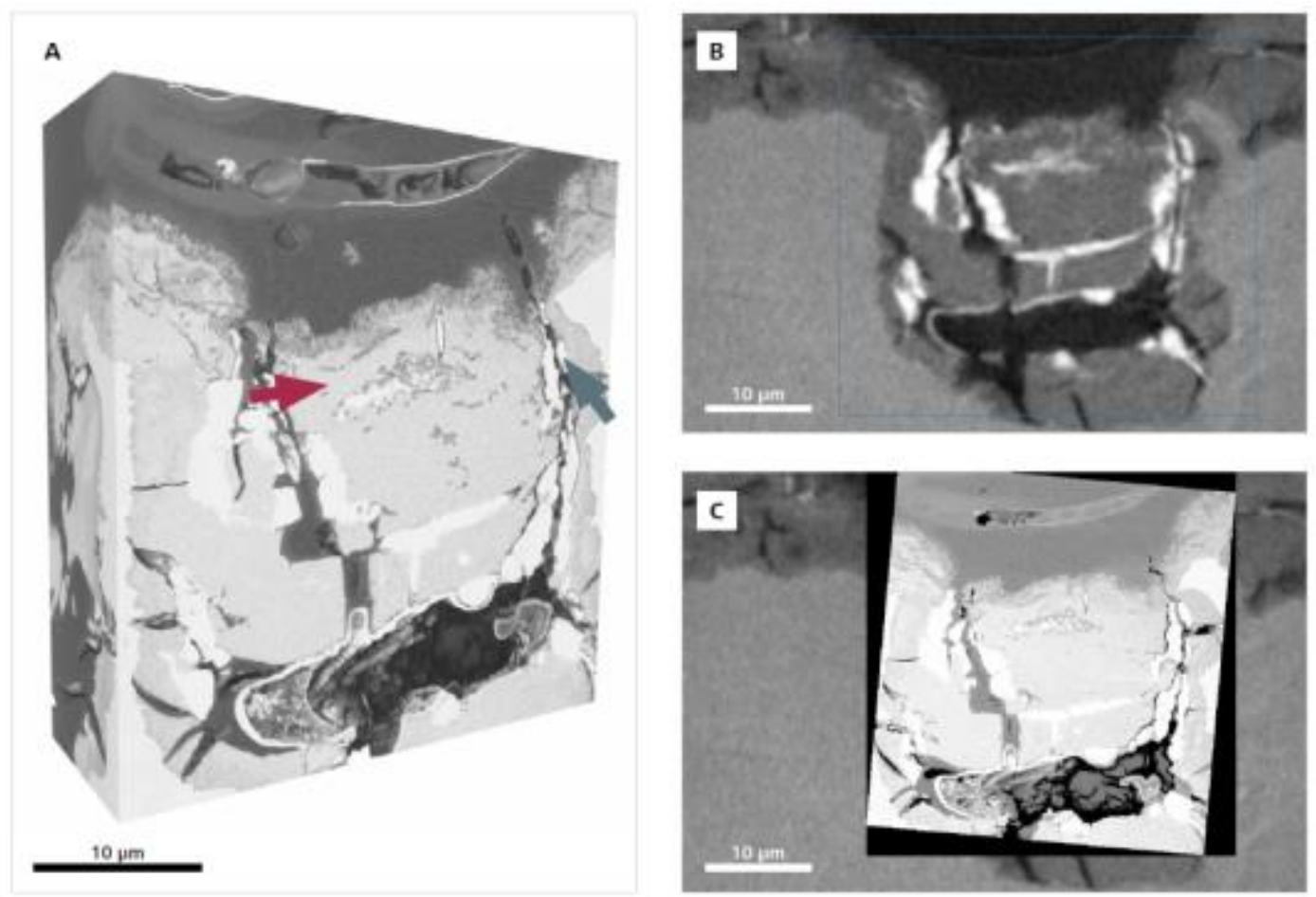

Figure 2. Correlative microscopy data on a corroded magnesium alloy. The specimen was monitored using submicron XRM as it was corroded in situ, from which an interesting region of corrosive attack was identified. This region was targeted and extracted using the FIB-SEM, after which it was imaged in 3D with the nano-scale XRM (top right). Finally, this same region was imaged with high-resolution FIB-SEM (left, bottom right). 